Itinéraires Itinéraires

Littérature, textes, cultures

\title{
Penser le corps en Islam
}

\section{Mustapha Ben Taïbi}

\section{OpenEdition}

\section{Journals}

Édition électronique

URL : http://journals.openedition.org/itineraires/1522

DOI : $10.4000 /$ itineraires. 1522

ISSN : 2427-920X

Éditeur

Pléiade

Édition imprimée

Date de publication : 1 novembre 2011

Pagination : 91-101

ISBN : 978-2-296-55720-8

ISSN : 2100-1340

Référence électronique

Mustapha Ben Taïbi, « Penser le corps en Islam », Itinéraires [En ligne], 2011-3 | 2011, mis en ligne le 01 novembre 2011, consulté le 02 mai 2019. URL : http://journals.openedition.org/itineraires/1522 ; DOI : 10.4000/itineraires. 1522

\section{(c) (1) $\Theta$}

Itinéraires est mis à disposition selon les termes de la licence Creative Commons Attribution - Pas d'Utilisation Commerciale - Pas de Modification 4.0 International. 


\title{
Penser le corps en Islam
}

\begin{abstract}
This article examines some ways of thinking the body in Islam. One direction would be to verify how "scholars" discourses describe the body: anthropologists and sociologists who have studied the social uses of the body; semiologists who described the body as a system of signs; psychoanalysts who highlighted the gap between the signifiers of desire and updates symptomatic and instinctual erotic philosophers who have sought to clarify the place of the body in the human world, not to mention the medical and ethical approach. But another difficulty for us to find our way in these discourses that go beyond our expertise is the paucity of studies that take the body as an object of reflection in the Arab and Muslim countries.
\end{abstract}

Keywords : body, Islam, linguistics, speech, discourse, tradition Mots clés : corps, Islam, sciences du langage, discours, tradition

J'ai des raisons de croire qu'on parle toujours « en tant que ». À tort ou à raison je suis considéré comme un linguiste (une linguistique du langage) et c'est à ce titre que j'aborde ici quelques façons de penser le corps en Islam. Ma position est interprétative, celle d'un lecteur pris dans l'esprit de son temps avec ses aspects multiples, ses contradictions, sa recherche d'une place entre les différentes façons de comprendre le fait religieux. En tout cas, il me semble que l'objet de cet article est une question de mise en mots par opposition à un savoir-faire rationnel ou technique par exemple.

Penser le corps en Islam renvoie à la rencontre avec les textes fondateurs. Le premier est le Coran qui évoque et donne à penser certains aspects du corps. Le deuxième correspond aux récits rapportés par la tradition qui forment la sunna (les dits, les faits et les gestes du prophète). On ne peut pas dire qu'avec ces deux ensembles de référence, nous avons une conception claire du corps en Islam, même si évidement la sunna propose son corps modèle, celui du prophète. Le troisième est le Kitâb al-naz̧ar fî ahkâm al-nazar bi-hâssat al-bașar de Abûl-Hasan 'Ali b. Muhammad b. al-Quattân al Fâsi, l'exemple même de l'effort (iztiâd) interprétatif fait par 
les théologiens pour mettre en place une certaine éthique du corps et des prescriptions juridiques.

Il n'est pas dans notre propos à cette occasion de nous interroger sur la « pensée » en général comme peut le suggérer le titre. Il s'agit plutôt pour nous de constater que tel texte présente le corps sous une forme surprenante qui nous « donne à penser », que tel « penseur » en présente tels aspects de façon banale, habituelle ou au contraire telle que nous ne la comprenons pas au premier abord, ce qui nous pousse à « penser » avec lui, mais autrement que lui. Avec cependant la possibilité de mettre entre parenthèses les discours qui posent des « essences » ou, en tout cas, de s'en méfier. Quand on parle de la « morale » ou de la « crise », tout le monde ne comprend pas de la même façon même s'il y a une part du commun et du générique qui s'impose dans chaque façon spécifique de comprendre. D'autant qu'il y a toujours là du plus ou moins facile ou difficile à dire ${ }^{1}$, sans, en tout cas qu'on puisse asserter que « tout » peut être dit. Il s'agit plutôt de poser la question du hors-langage, du contexte dans lequel les paroles ${ }^{2}$ prennent sens, mais aussi de ce qui résiste à la mise en mots. Quand on parle du langage et non pas de la langue, les difficultés à dire sont hétérogènes : les « trous de la langue », les censures, les tabous, les « énoncés sacralisés » et les « morales langagières », la minoration linguistique et la non-légitimation du locuteur, l'excès de pression, le conflit des interprétations.

À l'opposé, va se poser la question de ce qui pousse à « dire quand même ». Gardin décrit la situation du discours syndical ${ }^{3}$ opposé au discours patronal. Mais chacun de nous, j'imagine, a, par devers lui, des exemples de situations de conflits dans lesquels le « devoir dire » peut se manifester. Il ne s'agit pas donc pas d'une étude théologique sur le corps en Islam, ni de présenter un « état de la question ». Tout au plus, l'occasion nous est donnée, à travers cette thématique de continuer notre réflexion sur ce que c'est que s'orienter (et non que « savoir ») dans un champ compliqué et hétérogène qui est le discours religieux. Il s'agit de réflexion au sens de « retours sur », réflexion qui, tout en utilisant les outils des sciences du langage, demeure forcément marquée par la situation propre, le point de vue limité et en cela forcément biaisé de son auteur.

\section{Quand il y a prescription}

Le corps est appelé en arabe jasad, jisme, ou encore badan. Jasad désigne le corps que vient éveiller l'esprit, c'est donc le corps animé, la

1. Bernard Gardin, « Le dire difficile et le devoir dire », DRLAV, n 39, 1988, p. 1-20.

2. Il s'agit ici des paroles qui évoquent ou qui pensent le corps en Islam. De façon générale, il me semble que c'est une réflexion qui est nécessaire pour parler réellement du langage et plus spécifiquement des « genres du langage » effectifs et non pas de la langue.

3. Bernard Gardin, Langages et luttes sociales, éd. Nanon Gardin et Frédéric François, Limoges, Lambert-Lucas, 2005. 
chair. Le jisme désigne plutôt le corps comme substance, le corps extérieur. Enfin le badan désigne le corps volumineux, et notamment le tronc. Ce qui nous intéresse ici ce n'est pas le lexique en général, mais les différentes modalités de la « mise en mots » du « corps ». Ces différentes manières de dire sont autant de possibilités de faire effet, en particulier par la reprise du discours de l'autre, le déplacement, la modification. La référence d'un texte, ce dont il parle, se manifeste différemment selon qu'il s'agit de désigner une réalité absente ou une réalité présente. Avec le texte coranique, nous nous trouvons en présence d'une situation d' « inversion sémiotique » dans la mesure où la référence du texte coranique n'est pas représentable sur le modèle du doigt qui montre. Ceci dit, les modalités de la présence de cette absence se manifestent à travers l'évocation de quelques aspects, attitudes et expressions corporelles où s'opposent des notions de pur et d'impur : le processus de la création de l'homme, le rituel du jeûne, le ramadan, les interdits alimentaires, les pratiques funéraires, l'attitude du corps durant la prière et les rites de purification liés à la prière. Et puis il y a les versets relatifs à la question du hijâb et les versets qui concernent les comportements comme le regard. Sans omettre les récits qui évoquent ce qui attend les musulmans dans l'au-delà. Parfois, le texte est un peu plus explicite comme en ce qui concerne les pratiques alimentaires ou l'impureté qui provient de quatre aliments prohibés : la bête morte, le sang, la viande de porc et les victimes de sacrifices païens ${ }^{4}$. Toutes ces modalités différentes par lesquelles l'absent est en quelque façon présent renvoient à une question importante : celle de la façon dont cet absent retentit en nous, est ressenti. Ce sont les hadiths et la sunna qui vont définir les gestes et les pratiques à adopter dans les actes religieux cités ou les gestes de dévotion propres à l'islam. Une élaboration juridique qui permettra à la sunna de gonfler à loisir la liste des interdits. Ce qui relève du difficile à dire quand nous lisons ces textes, surtout le Coran et les hadiths, c'est leurs processus d'élaboration. De fait, il y a deux types de résistance au dire : celle de ce dont on parle que le discours ne rend jamais complètement transparent. Et puis, celle de l'effet du discours sur le récepteur, effet qui, lui aussi résiste plus au moins à une mise en mots adéquate. Et l'on pourrait ajouter ici, puisqu'on parle du Coran, du hadith et des traités de théologie, les difficultés spécifiques de l'approche de ces textes lointains relevant d'une culture lointaine dont les arrière-fonds nous échappent. Sachant bien qu'on ne peut pas dire grand-chose sur les conditions de production du Coran, et que ce qu'on peut analyser c'est surtout notre processus de lecture, on se demandera par exemple comment comprendre les trois versets suivants ${ }^{5}$ :

4. Hocine Ben Kheira, Islam et interdits alimentaires. Juguler l'animalité, Paris, PUF, 2000 .

5. Nous avons préféré citer les versets dans leur traduction française, laissons le soin aux lecteurs arabophones de se référer directement au Coran en arabe sans y ajouter une transcription phonétique. 


\title{
1) Sourate XXIV La lumière :
}

verset 30 : Dis aux croyants : de baisser leurs regards, d'être chastes. Ce sera plus pur pour eux. - Dieu est bien informé de ce qu'ils font-

verset 31 : Dis aux croyantes : de baisser leurs regards, d'être chastes, de ne montrer que l'extérieur de leurs atours (Blachère : agréments), de rabattre leurs voiles sur leurs poitrine, de ne montrer leurs atours qu'à leurs époux, ou à leurs pères, ou aux pères de leurs époux, ou à leurs fils, ou aux fils de leurs époux, ou à leurs frères, ou aux fils de leurs frères, ou aux fils de leurs sœurs ou à leurs servantes ou à leurs esclaves, ou à leurs serviteurs mâles incapables d'actes sexuels, ou aux garçons impubères.

Dis-leur encore de ne pas frapper (balchère : qu'elles ne piaffent pas) le sol de leurs pieds pour montrer leurs atours (blachère : agréments. Le mot est à entendre non seulement au sens des parures mais aussi des appas corporels) $[\ldots]$.

2) Sourate XXXVII, versets $40-49$ :

\begin{abstract}
À l'exception des serviteurs sincères de Dieu : à ceux là, comme un don bien connu, des fruits seront offerts. Ils seront couverts d'honneur dans les jardins du délice, placés sur des lits de repos se faisant vis-à-vis. On fera circuler une coupe remplie d'eau de source limpide et délicieuse... Celles qui ont des grands yeux et dont les regards sont chastes se tiendront près d'eux, semblables au blanc caché de l'œuf...
\end{abstract}

Premièrement, il me semble qu'il faut se résoudre à ne pas privilégier les versets comme seul niveau d'analyse du texte coranique. Il me semble en tout cas que l'approche par le biais des genres de discours est éclairante. Elle nous permet de préciser non la langue du texte mais les genres en tant que variations perpétuelles du mode d'action des discours sur ceux qui les reçoivent. La notion de genre n'est pas facile à manier. Il ne s'agit pas évidemment ici, d'une simple reprise de la classification traditionnelle des genres du type prose/poésie, épique/lyrique... On propose de distinguer les « grands genres » : poétique, religieux ${ }^{6} \ldots$ et $«$ genres locaux ${ }^{7} »:$ louange, ordre, blâme ${ }^{8} \ldots$

Ainsi, les versets 30 et 31 de la sourate XXIV sont de l'ordre du discours ${ }^{9}$. Ce sont donc des paroles de Dieu (un ordre : qul, dis) adressées à son prophète, son destinataire modèle premier, et à travers lui, à tous les destinataires modèles seconds dans une situation donnée. Pour autant,

6. Les grands genres peuvent se recouper : poétique et religieux.

7. Les genres de discours ne sont pas indépendants des différents types de thèmes et des conditions de discours.

8. Mustapha Ben Taibi, Quelques façons de lire le texte coranique, Limoges, LambertLucas, 2009.

9. On propose de définir le discours comme ce qui, oral ou écrit, fonctionne à un moment donné, et texte ce qui fonctionne à un autre moment, dans un autre contexte, le souvenir ou la perception par un tiers à qui le discours n'était pas destiné. 
peut-on dire que le sens de ce discours est inséparable de ses conditions d'énonciation? La relation du discours coranique à ses conditions d'énonciation n'est pas de l'ordre d'effet à cause. Le discours coranique ouvre un espace de « suspension » qui nous donne « un autre monde ». Ce qui n'exclut pas comme le montrent les trois versets cités, la possibilité de se référer à un espace pratique. Par contre le sens du contenu et la façon de signifier sont inséparables de l'affirmation de la notion de place ${ }^{10}$ assignable et légitime de celui qui parle. Il y a là une différence entre discours que chacun peut tenir et discours que seul Dieu et son prophète peuvent tenir. En tant que discours, le texte coranique suppose la possibilité de répondre à ce qu'il nous demande. Dans ce sens, on propose de considérer le langage en termes de dialogue et non d'énoncés " pris en eux-mêmes ». Les propos vont ainsi soit mettre en mouvement, enthousiasmer, indigner, interroger ou être accentués différemment par les uns et les autres. En fonction d'une part de l'esprit du temps, d'autre part de ses propres orientations, de façon explicite ou implicite. C'est dire que la possibilité d'une action en moi (en chacun de nous) peut être immédiate ou progressive sans que je me rende compte sur l'instant et sans que je sache (nous sachions) bien comment.

En tout cas, il y a là une certaine « force » qui agit sur nous ou plutôt la façon dont nous ressentons cette force et celle de la "représentation » abstraite que nous nous en donnons qui peut expliquer le sens que nous adoptons. Mais les rapports entre « sens » et « force » relèvent aussi du difficile à dire parce qu'il y a différentes figures du mot « force ». S'agit-il dans le cas du discours coranique de la contrainte ou de la « force » d'un interdit? De la force des énoncés eux-mêmes ou de la force de l'autorité de celui qui a dit que Dieu a dit? Mais il y a bien d'autres aspects de ce qu'on peut appeler « force », comme le choc de l'aspect merveilleux et/ou inquiétant de ce qui est révélé ou de ce qui « fait apparaître autrement » ${ }^{11}$.

Si les versets qu'on a cités sont de l'ordre du discours, les versets 40-49 de la sourate 37 sont de l'ordre du récit mythe. Tout mythe est réaccentuable. Quelles versions n'avons-nous pas entendues quand nous étions plus jeunes (le nous ici renvoie aux tous jeunes musulmans) de ce qui attend les bons ou les mauvais musulmans parmi nous? Le récit mythe ${ }^{12}$ se manifeste aussi comme «condensation », il se réduit au minimum. Et donc la beauté de la narration dans cet exemple ou la violence de la narration dans d'autres est entière. Et de ce fait le Coran nous dérange et nous donne à

10. On dira que la place de Dieu est unique. Celle de Mohammad en tant que prophète.

11. Sur les questions d'effet de texte, voir Frédéric François, Morales et mise en mots. Paris, L'Harmattan, 1994; Discours et entours. Essai sur l'interprétation, Paris, L'Harmattan, 1999. 12. On peut utiliser aussi l'expression récit mythique au lieu de récit mythe. L'essentiel est qu'on peut dire que le récit devient mythe lorsqu'il nous donne la figure concrète d'une réalité générique, la " chose même » en tant que dite, à la fois présente et absente. Ce mythe peut être familier ou constituer un choc. Il peut nous séduire ou nous contraindre quand il nous rappelle ce que nous avons tendance à oublier. 
penser. La lecture de ces versets au langage métaphorique nous fait vivre de possibles expériences dont le support est imaginaire mais qui sont pourtant immédiatement perceptibles. Les thèmes évoquent par leur présence dans le texte des entités réelles, mais cette valeur réaliste dépend moins des objets évoqués proprement dits que des sphères de représentation mentales et des conditions internes aux champs subjectifs de la perception.

Deuxièmement et pour revenir à la notion de contexte, les causes de la révélation (asbab al nouzul) sont loin de couvrir toutes les circonstances envisagées dans la situation du discours, d'autant plus qu'il s'agit souvent de sortes de petites histoires reconstruites pour chaque verset. Histoires par ailleurs recadrées, accentuées autrement et replacées selon les différentes situations. De plus, attribuer des fonctions aux versets peut être dangereux, dans la mesure où le présupposé retentit sur l'interprétation. Et puis « fonction » pour qui? Qui en sera juge? Ce qui revient à dire qu'il n'y pas un lien simple entre situation et discours. « On va donc ici du sens des paroles comme ne se comprenant que comme prises dans une situation, aux paroles donnant sens à la situation, à l'inversion de la notion de contexte ${ }^{13}$. »

\section{Le portrait du prophète}

On ne peut penser le corps en Islam sans parler de celui du prophète. C'est avant tout un homme, rien qu'un messager précise le Coran. Un homme aimant la vie, devenant prophète, animé par la foi absolue, l'espérance de résurrection dans le monde de l'au-delà.

Selon Ali, gendre du prophète et époux de Fatima-Zohra, Mohammed était de taille moyenne. Il avait un teint blanc-rosé, des yeux tout à fait noirs, des cheveux épais qu'il enduisait d'huile. Cette chevelure lui tombait sur les épaules. Ali a précisé que Mohammed avait un signe sur la poitrine...

D'autres détails : Mohammed n'avait pas de poils sur la partie inférieure du corps, une tête ronde de dimension moyenne. Une proportion harmonieuse des mains et des pieds. Un dos solide parsemé de poils. Il avait une démarche énergique, mais souple, empreinte de douceur et de modestie. Lorsqu'il avançait, il était précédé par une bonne odeur parfumée... Il se parfumait chez lui et lorsqu'il sortait ou rendait visite à ses compagnons, il se coupait les ongles et les enterrait selon les coutumes de l'époque. Il mettait sur ses yeux une sorte de Khôl (Kuhl), noir d'antimoine spécial. Il en mettait trois fois avant de s'endormir. Il se servait d'un cure-dents, jour et nuit. Parmi ses vêtements, tous consignés dans la tradition, c'est le manteau qui est le symbole de la sacralité, cité dans le Coran, sourate al-Muzammir, verset 1 : ô toi qui es enveloppé d'un manteau!

13. Frédéric François, « Discuter pourquoi faire? Ou la morale, le dire et le reste », dans R. Delamotte, Fr. François et L. Porcher (dir.), Langage. Éthique. Éducation, Rouen, Publications de l'université de Rouen, n² 231, 1997, p. 128. 
Les témoignages sur sa vie sexuelle et maritale sont plus au moins détaillés. D'après plusieurs paroles rapportées par la tradition, il visitait ses femmes le jour et la nuit... Il les visitait voilé, laissant tomber un pan de son vêtement sur la tête : « je n'ai jamais vu son sexe, confie Aïcha, et il n'a jamais vu le mien ». Mais Mohammed était à la fois un homme et un messager, un homme de désir et de prière, un stratège, le fondateur d'une nouvelle religion et d'une civilisation. La religion permet ainsi au corps d'assumer un statut social intériorisé, lequel s'adapte et évolue selon les coutumes et les mœurs de chaque communauté islamique. Mais qu'en est-il de l'image du corps prophétique dans le patrimoine islamique? Est-il interdit de représenter le visage du prophète? Oui, dans la mesure où, selon la tradition, le corps prophétique est marqué par des signes surnaturels qui font de Mohammed un homme et un messager. L'Islam à trouvé dans la calligraphie un moyen de régler ce dilemme en transposant l'image du visage dans le trait de l'écriture. Nous avons affaire ici à « des façons d'être humain » du prophète. Dans ce qui est rapporté par la tradition, il y a deux traits qui sont mis en avant. Il y a d'abord ce qui est partagé de fait : les caractères communs des êtres humains et les traits fréquents comme mode propre d'existence de sa propre communauté de l'époque. Il y a ensuite les données qui le différencient des autres et font de Mohammed un être « singulier ». Mais les manifestations corporelles du prophète (entre autres : les rêves et épreuves physiques qui ont précédé la révélation du Coran) ne sont jamais purement " sémiotiques ». Ensuite il est difficile de dire comment nous pouvons orienter notre compréhension de ce qui nous à été transmis parce qu'il y a à la fois ce qui nous rend possible de penser et ce qui nous empêche de penser, car ce qui constitue à un moment de «bonnes formulations », risque de devenir des formules toutes faites qui forment un prêt à penser. Le corps du prophète reste énigmatique.

Après le corps du prophète il y a celui du saint. C'est un corps bénéfique. Le saint a la baraka, déjà observée chez le prophète. Il guérit par imposition des mains, par application d'un crachat, par relique de contact ayant touché son corps... Les critères de sainteté sont entre autres : jeûnes miraculeux, veilles prolongées, don de larmes, avilissement d'un corps méprisé, ascèse poussée... On peut aussi parler du saint choquant, le majdhub au Maghreb ou ailleurs. On peut aussi d'une certaine façon parler de la sainteté des princes ou rois liée à la sacralité du pouvoir...

\section{Quand il y a jurisprudence}

L'élaboration juridique des pratiques et gestes du corps à adopter revient pour l'essentiel à la sunna et aux différents théologiens des différentes écoles musulmanes. Un des textes les plus exhaustifs en la matière est celui d'Abu l-Hasan Ali. Muhammad b. al-Quattan al-Fasi (1130-1269). Son traité sur le corps «montré » et le corps « regardé » selon la loi divine, 
le Kitâb. al-nazar fi ahkami al-nazar bi-hâssat al-bașar ${ }^{14}$ se base sur les versets 30-31 déjà cités de la sourate XXIV. Cet ouvrage est composé de huit chapitres :

1. Du fait que baisser le regard est prescrit par la loi

2. Exposé de ce qu'il est permis de laisser apparaître au regard et ce qui n'est pas permis

3. Du regard des hommes sur les hommes

4. Du regard des femmes sur les femmes

5. Du regard des hommes sur les femmes

6. Du regard des femmes sur les hommes

7. Des nécessités «mitigentes » (fi l'darûra al-mubiha)

8. Exposé du fait qu'il est proscrit par la loi de prononcer tels dires ou d'accomplir tels actes à la vue de certaines choses

Ibn al-Quattân accorde à la notion de 'awra cité dans le verset 31, qui désigne généralement les parties du corps d'une personne qu'elle ne peut dévoiler ou qu'autrui ne peut voir, une importance capitale. Mais cette notion n'est point explicite dans le Coran, comme c'est souvent le cas en matière de normativité, tout au plus il parle de 'awrat al nisa' " awrat des femmes » sans description. Reste que, malgré tout, pour qu'il y ait interprétation, il faut qu'il y ait incertitude et non certitude et complète transparence. C'est ce que permet le texte coranique et c'est ce dont va se saisir al Quattan pour préciser son point de vue sur la notion d' 'awra: « ce dont l'apparition sème le trouble ${ }^{15} »$. Il serait trop long de s'arrêter sur chaque question traitée (pas moins de 171) ou chaque chapitre ${ }^{16}$. Prenons juste deux exemples :

\section{Le point de vue du / de la regardé-e}

En réponse à la question « qu'est-il permis de laisser apparaître de son corps aux regardants? », Ibn al-Quattân distingue dans la communauté trois classes : les hommes, les femmes et les hermaphrodites.

Pour les êtres de sexe masculin (al-dhukûr), Ibn al-Quattân fait la part entre ceux d'entre eux qui sont « obligés devant la loi » et ceux qui ne le sont pas, c'est-à-dire les garçons impubères. Pour les obligés, ils doivent laisser apparaître de leurs corps ce qui est au-dessus du nombril et ce qui

14. Ibn al-Qațtân al-Fâsi, éd. 'Ali ibn Muhammad, Idrīs Șamadî et Fârûq Ḥamâdah, Kitâb

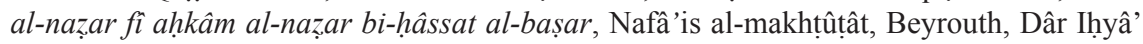
al-'Ulûm, 1996.

15. Ibn al-Qattân al-Fâsi, op. cit., p. 116, p. 310.

16. Pour une analyse plus détaillée des parties de cet ouvrage, voir Éric Chaumont, « La notion de 'awra selon Abû l-Hasan 'Ali b. Muhammad b. al-Quttân al-Fâsi (m. 628/1231) ", Revue des mondes musulmans et de la Méditerranée, $\mathrm{n}^{\circ}$ 113-114, 2006, p. 109-123, [En ligne] http://remmm.revues.org/index4052.html. 
est au-dessous du genou. La notion de 'awra ici couvre les saw'atayn, soit l'anus et la verge. Pour les seconds, ils ne sont pas concernés par la loi.

Pour des êtres de sexe féminin la même distinction est établie : d'un côté les jeunes filles impubères non assujetties à la loi et de l'autre les femmes nubiles obligées devant la loi. En ce qui concerne ces dernières, la détermination de la 'awra de la femme se fait en considération de la notion de zîna « atours » (la sourate XXIV, 31) et c'est là que réside le problème. Les discussions autour de cette question, à savoir les « atours » que la femme ne peut montrer qu'aux personnes évoquées dans le verset n'ont pas abouti à un consensus (ijma'). Parfois il est question du visage de la femme (alwajh), pour d'autres se sont les mains (al-kaffayn) et/ou les pieds (al-kadamayn). La voix de la femme est en elle-même une 'awra à dissimuler.

\section{Le point de vue du/de la regardant-e}

La touche subjective d'Ibn-al-Quattân se manifeste dans l'étude de différentes situations particulières où c'est l'intentionnalité du regard qui est jugée. Ainsi par exemple, le regard d'un homme sur le garçon impubère est autorisé puisqu'il n'est pas dans l'obligation de couvrir sa nudité. Mais si ce regard est la jouissance (al-taladhdhudh) ou la «crainte d'être éprouvé (khawf al-iftitân), le corps entier du jeune homme est interdit. Le principe est le même concernant le statut du regard sensuel porté par une femme sur une autre femme, celui d'un homme sur une femme ou d'une femme sur un homme. La même injonction est de mise : « baisser les yeux devant tout ce dont la vue éveille la passion est obligatoire ${ }^{17} \gg$.

Comment comprendre ce texte qui est représentatif d'une longue tradition?

On peut mettre en parallèle deux niveaux de réflexion : le premier niveau est relatif au savoir objectif-philologique qui est mis en avant dans l'interprétation du Coran, aux autres auteurs de son temps ou avant lui qui ont traité cette problématique. On a déjà signalé que la signification passe par le langage et la mise en mots et que cette question du signifié dans la langue est aussi ambiguë que celle de la compréhension comme reconstitution de l'intention de l'auteur. La manière dont les exégètes interprètent les versets 30-31 de la Sourate XXIV, surtout la définition des notions de zina « atours » et 'awra est un exemple en la matière ${ }^{18}$. Il faut plutôt parler

17. Ibn al-Qattân al-Fâsi, op. cit., p. 353.

18. Tabari par exemple souligne trois exégèses : les atours qui ne doivent pas être montrés (les bracelets que l'on met aux bras et aux pieds, les boucles d'oreilles, les colliers) et ce qui ne peut être caché, parce que nécessairement apparent (les habits) (X, XVIII, 117); les atours apparents (zîna žahira), qu'il est donc licite de découvrir, sont d'après Ibn 'Abbas (m. 68/686 ou 688) : «le visage, le fard des yeux, le henné des mains et les bagues; mais la femme ne peut les découvrir que chez elle, quand elle reçoit des gens » (ibid., p. 118); la 
ici de pratiques sociales qui visent à l'identification du signifié comme sens déterminable dans la langue. Le deuxième niveau peut être formulé de cette façon : que peut signifier ce discours pour nous? On peut répondre de plusieurs manières.

On peut, d'une certaine façon, dire que les hommes de cette époque « pensaient comme ça ». Un point c'est tout. Mais je ne pense pas qu'on puisse renvoyer les idées de cet auteur à un passé révolu.

On peut reconstituer les conditions de ce discours qui fait sens dans son milieu autrement que dans le nôtre ${ }^{19}$. Mais en religion comme en droit et en tout temps, la conviction que les décisions pratiques doivent s'appuyer sur des règles claires exerce une forte fascination sur les gens. Reste que dans la vie quotidienne, le point de vue théorique religieux est une chose, le traitement effectif des cas concrets particuliers en est une autre. Un peu comme la différence entre ce que peut nous dire un discours conceptuel sur nos façons de faire ou d'être et ce qu'une expérience romanesque peut apporter comme éclairage sur différentes façons d'exister et de faire sens. Finalement la question avec les discours qui posent des essences ce n'est pas de savoir si les règles ou les notions qu'ils développent sont « vraies » ou « justes », mais ce qu'ils nous aident à percevoir et ce qu'ils nous font éventuellement négliger.

Pour penser le corps en Islam de façon exhaustive, il faut aller à la rencontre d'autres textes appartenant à la littérature de hadith (corpus et commentaires) et à la littérature exégétique dans son ensemble, chi'ite comprise, même si, par ailleurs, on peut considérer que leurs oppositions importent plus que leurs ressemblances. En ce sens, il faut insister sur les autres figures de la pensée en mettant en avant la diversité des genres de discours sur le sujet : celui de l'anthropologue, du sociologue et du psychanalyste. De même chez les anciens, des théologiens (ils étaient à la fois juristes, grammairiens et linguistes) qui ne manquaient pas d'ardeur religieuse, ont développé une littérature de la courtoisie, de l'aimance et de l'amour qui a couvert mille ans, jusqu'au XVI ${ }^{e}$ siècle, à l'image d'Ibn Hazm (993-1064), auteur du Collier de la colombe ${ }^{20}$. À tout genre de discours correspond un type de pensée ou plusieurs. Et parmi la multitude

troisième exégèse attribuée à Hasan l-nasri (m. 110/728) : « les atours que la femme peut dévoiler sans crainte sont le visage et les habits » (ibid., p. 119).

19. «L'un des thèmes favoris de la propagande almohade avait partie liée avec une question revenue à l'ordre du jour - et de quelle manière! - : la question du voilement, ou, de manière à la fois plus précise et plus générale, celle de la Loi ou de l'éthique vestimentaire musulmane... Abûl-Hasan'Ali b. Muhammad b. al-Quattân al Fâsî (562/1167-628/1231) était un "intellectuel organique", un 'âlim, un savan dont toute l'activité et le statut même étaient placés sous le signe de la promotion de l'idéologie et du pouvoir almohades » (Éric Chaumon, op. cit., p. 110).

20. Ibn Hazm, Le Collier de la colombe, trad. Gabriel Martinez-Gros, Arles, Actes Sud, 2009. 
des textes qui évoquent ou pensent le corps en Islam ${ }^{21}$, c'est peut-être le texte narratif qui éclaire le mieux ce qui se vit « pour de vrai ». C'est dans le récit qu'apparait « la distance entre le su, le dit, la façon de vivre, l'aléa des décisions, les retournements qui font que ce qui a été décidé a des conséquences inattendues ». Et c'est le récit « qui va pouvoir manifester la distance entre les principes que nous affirmons et/ou les lois générales "d'explication des comportements humains" et les particularités de l'événement, de ses conséquences et de la diversité des façons de les percevoir ${ }^{22}{ }^{2}$.

\author{
Mustapha Ben Taïbi \\ Université Paris Descartes - Faculté des Métiers, Evry
}

21. Mais aussi de façon générale.

22. Frédéric François, Essais sur quelques figures de l'orientation, Limoges, LambertLucas, 2009, p. 127. 